\title{
Aromatase inhibitors, efficacy and metabolic risk in the treatment of postmenopausal women with early breast cancer
}

\author{
Stefano Gonnelli ${ }^{1}$ \\ Roberto Petrioli ${ }^{2}$ \\ 'Department of Internal Medicine, \\ Endocrine-Metabolic Science and \\ Biochemistry, University of Siena, Italy \\ (Dir. R. Nuti.); ${ }^{2}$ Department of Human \\ Pathology and Oncology, Medical \\ Oncology Section, University of Siena, \\ Italy (Dir. G. Francini)
}

\begin{abstract}
The third-generation aromatase inhibitors (AIs), letrozole, anastrozole and exemestane, are becoming the first choice endocrine drugs for post-menopausal women with breast cancer, since they present greater efficacy when compared with tamoxifen in both adjuvant and metastatic setting. In particular, several large and well designed trials have suggested an important role for AIs in the adjuvant treatment of postmenopausal women with estrogen-receptor positive breast cancer either in the upfront, sequential or extended adjuvant mode. Overall, AIs are associated with a small but significant improvement in disease free survival. The expanding use of AIs in the treatment of early breast cancer means that individual patients will be exposed to the agents for longer durations, making it increasingly important to establish their long-term safety. This review focused on the effects of AIs on bone metabolism, serum lipids and cardiovascular risk. AIs have adverse effects on bone turnover with a reduction of bone mineral density and an increase in the rate of fragility fractures. With respect to tamoxifen AIs present lower thrombotic risk and a less favorable impact on lipid profile, whereas the true effects on cardiovascular risk still remain to be clarified. An adequate monitoring of bone mineral density (BMD) and lipid profile could be recommended for post-menopausal women candidate to AIs.
\end{abstract}

Keywords: breast cancer, aromatase inhibitors, bone loss, lipids, cardiovascular risk

\section{Efficacy of aromatase inhibitors}

The third-generation aromatase inhibitors (AIs), letrozole, anastrozole and exemestane, are now widely accepted as alternatives to tamoxifen as first-line therapy in postmenopausal women with estrogen receptor (ER)-positive advanced breast cancer, because of their improved clinical effectiveness (Nabholtz et al 2000; Bonneterre et al 2001; Mouridsen et al 2001). Despite the fact that tamoxifen remains an effective drug, AIs appear to be superior to this agent as first-line endocrine therapy for metastatic breast cancer also according to a pooled analysis of 8 randomized studies (Carlini et al 2005).

The favorable efficacy and safety profile in the advanced disease has encouraged the evaluation of third-generation AIs in the adjuvant setting. Several phase III randomized, adjuvant trials have assessed third-generation AIs in comparison with tamoxifen or placebo after 5 years or less of tamoxifen therapy. The results of these studies in terms of disease-free survival are summarized in Table 1.

The BIG 1-98 (Big International Group) trial addressed whether letrozole in the treatment of postmenopausal women with ER positive breast cancer is more effective if used as an initial adjuvant therapy or as sequential therapy following adjuvant tamoxifen (Thurlimann et al 2005). Five years after randomization, $84.0 \%$ of patients in the letrozole group and $81.4 \%$ in the tamoxifen group were disease-free, corresponding to a $19 \%$ relative or $2.9 \%$ absolute treatment difference. The absolute reduction in 
Table I Efficacy of aromatase inhibitors (Als) expressed as disease free survival in the adjuvant setting

\begin{tabular}{llllll}
\hline $\begin{array}{l}\text { Treatment } \\
\text { strategy }\end{array}$ & Trial & Protocol & $\begin{array}{l}\text { Follow-up } \\
\text { (months) }\end{array}$ & $\begin{array}{l}\text { Relative risk } \\
\text { reduction (\%) }\end{array}$ & $\begin{array}{l}\text { Absolute risk } \\
\text { reduction (\%) }\end{array}$ \\
\hline Upfront & ATAC & Ana vs Tam & 100 & 15 & 4.1 \\
& BIG I-98 & Let vs Tam & 51 & 19 & 2.9 \\
Sequential & IES & Tam $\rightarrow$ Exe vs Tam & 55.7 & 24 & 3.3 \\
& ARNO & Tam $\rightarrow$ Ana vs Tam & 30.1 & 34 & 4.2 \\
& ABCSG/ARNO & Tam $\rightarrow$ Ana vs Tam & 28 & 40 & 3.1 \\
Extended adjuvant & ITA & Tam $\rightarrow$ Ana vs Tam & 64 & 44 & 7.9 \\
\hline
\end{tabular}

Abbreviations: Ana, anastrazole; Tam, tamoxifen; Let, letrozole; Exe, exemestane; ATAC, anastrazole, tamoxifen alone or in combination (ATAC Trialists' Group 2008); BIG, breast international group (Coates et al 2007); IES, intergroup exemestane study (Coombes et al 2007);ARNO, arimidex-nolvadex (Kaufmann et al 2007); ABCSG, austrian breast cancer study group (Jakesz et al 2005); ITA, italian tamoxifen anastrazole (Boccardo et al 2006); MA I7, (Goss el al 2005).

cumulative breast cancer relapses also significantly favored letrozole over tamoxifen at the fifth year (10.2\% vs $13.6 \%$, $\mathrm{p}=0.0002$ ) (Thurlimann et al 2005; Coates et al 2007).

The ATAC (Arimidex, Tamoxifen Alone or in Combination) trial compared adjuvant anastrozole with tamoxifen: more than 9,000 postmenopausal women were randomly assigned to receive anastrozole plus placebo, or tamoxifen plus placebo, or anastrozole plus tamoxifen. With a median follow-up of 47 months, in comparison to the tamoxifen arm, anastrozole resulted in a statistically significant reduction in breast cancer events and an improvement in disease free survival (Baum et al 2002).

After completion of 5 years' adjuvant treatment anastrozole significantly prolonged disease-free survival and significantly reduced distant metastases and controlateral breast cancers (ATAC Trialists' Group 2005). Recently the ATAC Trialists' Group has reported findings from an analysis of 100-month follow-up data. This analysis showed that in hormone-receptor positive populations the improvement in disease control with anastrozole with respect to tamoxifen was maintained for over three years after treatment cessation (ATAC Trialists' Group 2008).

The Intergroup Exemestane Study (IES) randomly assigned 4742 women who had received 2 to 3 years of tamoxifen to continue tamoxifen for a total of 5 years or to receive exemestane in order to complete a 5-year course of hormonal therapy. After a median follow-up of 55.7 months, the trial demonstrated a significant reduction in events (recurrence, contralateral breast cancer, or death) in favor of the exemestane arm (Coombes et al 2007).

In the Italian Tamoxifen Anastrazole (ITA) study, women who had received 2 to 3 years of tamoxifen were randomly assigned to either continue treatment with tamoxifen for a full 5 years or to receive anastrozole. A total of
448 patients were enrolled: at a median follow-up time of 64 months, 63 events had been reported in the tamoxifen group compared with 39 in the anastrozole group (HR 0.57, 95\% CI 0.38-0.85, p =0.005). Relapse-free and overall survival were also significantly longer in the anastrozole group (Boccardo et al 2006).

The ARNO (ARimidex-Nolvadex) study reported that postmenopausal women who have taken tamoxifen for 2 years as adjuvant therapy are less likely to experience a recurrence of breast cancer and have an improved possibility of survival if they switch to anastrozole (Kaufmann et al 2007).

A combined analysis of data from two randomized trials with almost identical inclusion criteria was performed in the ABCSG/ARNO study. Postmenopausal women who had completed 2 adjuvant years of oral tamoxifen (20 or $30 \mathrm{mg}$ daily) were randomized to receive $1 \mathrm{mg}$ oral anastrozole or tamoxifen ( 20 or $30 \mathrm{mg}$ daily) for the remainder of their adjuvant therapy. A total of 3224 patients were included in the analyses: at a median follow-up of 28 months, there was a $40 \%$ decrease in the risk of an event in the anastrozole group as compared with the tamoxifen group (HR $0.60,95 \%$ CI $0.44-0.81, p=0.0008$ ) (Jakesz et al 2005).

The MA-17 trial randomly assigned postmenopausal women who were completing 5 years of tamoxifen treatment, to receive either letrozole or a placebo for an additional 5 years after adjuvant tamoxifen. After a median follow-up of 2.4 years, the study was halted by the Data Safety Monitoring Board because of significant reduction in breast cancer events in the letrozole group (Goss et al 2005).

Similar results have been reported in the analysis of the Austrian Breast Colorectal Cancer Study Group (ABCSG)-6a open label trial of extended adjuvant therapy with anastrozole for 3 years after completion of 5 years of adjuvant tamoxifen, with or without aminoglutethimide. 
At a medium follow-up of 62.3 months the women who received anastrozole had a significantly reduced risk of recurrence compared with women who received no further treatment (Jakesz et al 2007).

A recent meta-analysis evaluated the benefit in event-free and overall survival of AIs after 2 to 3 years of tamoxifen, and revealed that the early switch strategy improves survival over the standard tamoxifen 5-year treatment. The risk of any event is reduced with AIs by $23 \%$, with an absolute benefit of $3.8 \%$ (Bria et al 2006).

Therefore, the available data from randomized adjuvant trials in early breast cancer recommend that the optimal adjuvant treatment for postmenopausal women with early breast cancer should now include AIs as either initial therapy or sequential therapy after tamoxifen treatment. Recent modeling data suggest that using AIs as upfront adjuvant treatment is better than using AIs in sequence after 2 or more years of tamoxifen (Cuzick et al 2003).

Finally when used as preoperative (neoadjuvant) treatment, AIs showed greater efficacy than tamoxifen with regard to breast conservation rate but not to clinical response rate (Cataliotti et al 2006).

\section{Mechanism of action of Als and other hormone therapies for breast cancer}

A comprehensive description of the mechanism of action of hormone therapies for breast cancer is present in two recent reviews (Jordan 2007; Journè et al 2008).

Although tamoxifen is an inhibitor of breast cancer growth, its effects throughout the human body vary and could be characterized as having mixed estrogenic properties. Most of the estrogenic properties are desirable, eg, preservation of bone mineral density in postmenopausal women or decreases of low-density lipoproteins, but these partial estrogen agonist effects may also be detrimental and a likely cause of the increased risk of some toxicities such as thromboembolic events and endometrial cancer (Ganz 2001). In contrast, fulvestrant is a new estrogen-receptor antagonist, with no known agonist (estrogenic) effects, which has recently been licensed for treatment of advanced breast cancer in postmenopausal women (Journé et al 2008). Recent studies have reported that about $30 \%$ of postmenopausal women with advanced breast cancer who had progressed following prior antiestrogen therapy, gained clinical benefit with fulvestrant therapy thus delaying the need for chemotherapy (Journé et al 2008; Neven et al 2008). Available data suggest that fulvestrant, given intermusculary, is well tolerated, with a low incidence of treatment-related adverse events and injection-site reactions (Neven et al 2008).

Another recent endocrine treatment is represented by third-generation AIs which block the estrogen receptor by reducing the levels of its ligand, endogenous estrogen. They target the aromatase enzyme (a P-450 cytochrome enzyme), which converts testosterone and adrenal androgens to estradiol and other estrogens (Smith and Dowsett 2003).

AIs are categorized in two types, non-steroidal and steroidal, and differ in their modes of interaction with the aromatase-enzymatic complex and its inactivation. Non-steroidal AIs, anastrozole and letrozole, are imidazole-based and compete with endogenous substrates for access to the cytochrome P-450 moiety of aromatase, where they form a reversible co-ordinate bond. The steroidal AI exemestane is an analogue of androgens which competes with the endogenous androstenedione and testosterone for access to the cytochrome P450 moiety of aromatase and causes irreversible enzyme inhibition.

Molecular differences between anastrozole, letrozole and exemestane affect their selectivity for the aromatase enzyme and thus their capability in the inhibition of total-bodyaromatization and, as a result, plasma estrogen suppression (Budzar and Howel 2001; Smith and Dowsett 2003).

These differences among the different AIs may explain the partial non-cross-resistance between steroidal and non steroidal AIs which allows the possibility of using exemestane after non-steroidal AIs (Ponzone et al 2008).

The shift from tamoxifen to an AI in both the advanced and adjuvant setting has challenged the status of tamoxifen as the 'gold standard' treatment for postmenopausal women with hormone-receptor-posistive breast cancer. Therefore, the expanding use of AIs in the treatment of early breast cancer means that individual patients will be exposed to these agents for longer durations, making it increasingly important to establish their long-term safety. The aim of this review was to focus on the possible different effects of tamoxifen and AIs on bone metabolism, lipid profile, and cardiovascular disease in postmenopausal patients with early breast cancer.

\section{Effects of Als on bone metabolism}

Estrogens play a crucial role in maintaining both normal bone turnover and normal bone mass, and therefore long-term estrogen deprivation may be associated with the development of osteoporosis and increased susceptibility to bone fractures. The better efficacy of AIs with respect to tamoxifen in breast cancer patients is usually explained by the fact that they reduce peripheral estrogen concentrations to extremely low levels, 
while tamoxifen has partial agonist activity. The profound estrogen suppression achievable with the third-generation AIs could also explain some unfavorable effects on bone (Brufsky 2008). Nevertheless, it has been reported that the reduction of the low residual levels of serum estradiol to virtually undetectable levels in healthy late postmenopausal women is associated with further increases in bone resorption rate (Heshmati et al 2002). Moreover, the lower levels of serum estradiol are associated with the higher rates of bone loss and the greater risk of fractures in late postmenopausal women (Cummings et al 1998). The complete estrogen deprivation may lead to an increased production of the cytokine RANKL by stromal cells and increased activity of RANK, thus leading to increased numbers of osteoclastic precursors and osteoclastogenesis. This, along with the reduction in circulating levels of osteoprotegerin, results in an increase in the number of mature osteoclasts and consequently increased bone breakdown (McCloskey 2006).

Despite the effects of the third generation AIs on bone turnover having been reported in several studies, it is difficult to assess the difference between the three available agents in the absence of head-to-head comparisons. A small study performed in postmenopausal women with advanced breast cancer described significant increases in markers of bone formation and bone resorption after 3 months of anastrozole (Bajetta et al 2002).

The bone substudy of the ATAC trial has reported that postmenopausal women with early breast cancer, treated for 5 years with anastrozole, presented losses of bone mineral density (BMD) both at lumbar spine and total hip, at year $1(-2.3 \%$ and $-1.5 \%$, respectively) at year $2(-4.0 \%$ and $-3.9 \%$, respectively) and at year $5(-6.0 \%$ and $-7.2 \%$, respectively) (Eastell et al 2008). On the other hand the treatment with tamoxifen was associated to significant increases in both lumbar spine BMD (1.4\% at year 1, 2.1\% at year 2 and 2.8 at year 5) and total hip BMD (0.8\% at year $1,1.2 \%$ at year 2 and $0.7 \%$ at year 5) (Eastell et al 2008). In this study one-year treatment with anastrazole significantly increased both bone formation markers (bone alkaline phosphatase [bone-ALP] $20 \%$ and procollagen type-I N terminal propeptide [PNP] 18\% respectively) and bone resorption markers ( $\mathrm{C}$-telopeptide of type-I collagen [CTX] 26\% and N-telopetide of type-I collagen [NTX] 15\% respectively). On the contrary tamoxifen induced a marked decrease in both bone resorption markers (CTX: $-56 \%$ and NTX: $-52 \%$ ) and bone formation markers (PINP: $-72 \%$ and bone ALP: $-16 \%$ ). The withdrawal of tamoxifen was followed by a tendency of bone markers to return towards baseline values in a period of time ranging from a few weeks to several months (Eastell et al 2006).

In the adjuvant ATAC trial, although the anastrozole safety profile was better than that of tamoxifen overall, there was an incidence of fractures significantly higher in the anastrozole arm as compared to that in the tamoxifen arm (11.0\% vs 7\%) after a median follow-up of approximately 33 months (Baum et al 2002).

A recent article by the ATAC Trialists' Group has reported the findings from an analysis of 100-month follow-up data. This analysis showed that fracture rates were higher in patients receiving anastrozole than in those receiving tamoxifen during active treatment [incidence rate ratio (IRR) 1.55 (1.31-1.83), $\mathrm{p}<0.001]$, but were not different after treatment was completed [IRR $=1.03(0.81-1.31), \mathrm{p}=0.79$ ]. Therefore, the increase in fracture rates with anastrozole seems to be associated only with the active treatment period and does not continue after its completion (ATAC Trialists Group 2008).

Assuming a baseline annual fracture rate of 17 fractures per 1000 healthy postmenopausal women, survivors of breast cancer not treated with adjuvant hormonal therapy have a relative fracture risk of 1.15 (20 fractures per 1000 women). Based on the ATAC trial, patients with breast cancer treated with anastrozole have a relative fracture risk of 1.36 (23 fractures per 1000 women). This means 2 additional fractures per year in 300 postmenopausal women with early breast cancer. In contrast, patients treated with tamoxifen have a relative fracture risk of only 0.91 , suggesting that tamoxifen may have bone-protective effects (Brufsky 2008).

The Italian Tamoxifen Anastrozole (ITA) study suggested no statistically significant difference in fracture risk after a median follow-up of 36 months between the postmenopausal women with early breast cancer switched from tamoxifen to anastrozole and those who continued taking tamoxifen (Boccardo et al 2006).

In the MA-17 trial, at a median follow-up of 1.9 years there were more cases of newly diagnosed osteoporosis in the letrozole group compared with the placebo group (5.8\% vs $4.5 \%$ ); fractures were more frequent in the letrozole group $(3.6 \%$ vs $2.9 \%$ ), but the difference was not statistically significant. Since MA-17 underwent an early termination because of the positive results of disease-free survival, some questions regarding the long term effects of letrozole on bone remain unanswered (Goss et al 2005).

Letrozole has also recently been shown to result in significantly greater loss of BMD at total hip $(-3.6 \% \mathrm{vs}-0.71 \%$ for placebo; $\mathrm{p}=0.44)$ and lumbar spine $(-5.3 \% \mathrm{vs}-0.70 \%$ for 
placebo; $p=0.008$ ) after 2 years of treatment in patients with breast cancer who had previously received 5 years of adjuvant tamoxifen therapy (Perez et al 2006). Moreover, the results of the BIG 1-98 study indicate a statistically significant increase in fracture number in the letrozole arm with respect to the tamoxifen arm (9\% vs 6\%) (Viale et al 2007).

On the basis of experimental studies exemestane, due to its steroidal structure, was expected to be less detrimental to bone with respect to the non-steroidal anastrozole and letrozole. The effects of 24 months of treatment with exemestane, anastrozole and letrozole on the serum and urine levels of markers of bone turnover were recently compared in 84 healthy postmenopausal women. Bone resorption markers, such as NTX, were increased to the greatest extent by letrozole, while exemestane was associated with increased serum levels of the bone formation marker serum PINP (Goss et al 2007). This finding suggests a specific bone formation effect of exemestane with respect to other Ais, and this was attribuited to a possible androgenic activity of exemestane.

A study carried out in postmenopausal women with early breast cancer demonstrated that a 2-year treatment with exemestane significantly increased both the markers of bone resorption (CTX: 35\% and NTX: 13.7\%) and of bone formation (PINP 44.1\% and bone-ALP 51.7\%) (Lonning et al 2005). Exemestane induced a non significant increase in the mean annual rate of BMD loss at lumbar spine with respect to placebo $(2.17 \%$ vs $1.84 \%)$ and a slightly significant increase in femoral neck BMD (2.72\% vs $1.48 \%$; $\mathrm{p}=0.02$ ) (Lonning et al 2005).

Two recent reports described that the switching of postmenopausal women with early breast cancer from tamoxifen to exemestane causes a marked increase in bone turnover markers with a consequent reduction in BMD (Gonnelli et al 2007; Coleman et al 2007). In the first study, at the end of 1 -year treatment with exemestane, the increase in bone-ALP was 31.5\%, that of CTX was 105.4\%and the decrease in BMD parameters was significant both at lumbar spine $(-2.37 \%, \mathrm{p}=0.05)$ and at femoral neck $(-1.24 \%, \mathrm{p}=0.05)$ (Gonnelli et al 2007). The larger IES study described that in the first 6 months after the switching from tamoxifen to exemestane BMD decreased by $2.7 \%$ at lumbar spine and by $1.4 \%$ at total hip. After a median follow-up of 58 months and median exposure to exemestane of 30 months, 162 (7\%) patients in the exemesane group had fractures, compared with $115(5 \%)$ patients in the tamoxifen group (OR: 1.45; p = 0.003) (Coleman et al 2007). Despite its steroidal structure and putative androgenic activity, these data confirm that, similarly to other AIs, exemestane exerts a negative effect on bone.
Bone loss and fracture risk were also analyzed retrospectively in a patient-claims database of women with early-stage breast cancer and no osteoporosis who received an AI $(n=1.354)$ and those who did not $(n=11.014)$. The prevalence of osteoporosis was $8.7 \%$ in the AI group versus $7.1 \%$ in the control group $(\mathrm{p}=0.01)$. Also, the risks for both bone loss and fracture were significantly higher in the AI group than in the control group (27\% and $21 \%$ respectively; $\mathrm{p}=0.02$ ) (Mincey et al 2006).

Therefore, current data suggest that all the third-generation AIs have adverse effects on bone. Also, a recent meta-analysis showed that bone fractures were significantly higher in patients receiving AIs than in those who did not (HR 1.50, 95\% CI 1.12-2.02, $\mathrm{p}=0.006$ ) (Bria et al 2006). However, because of the lack of comparative data, the drawing of any conclusions on any clinically relevant differences between these agents is difficult. Given the expanding use of AIs, a diffused screening, an appropriate monitoring and adequate treatment strategies are necessary for women candidate to AIs. The American Society of Clinical Oncology (ASCO) recommends that the breast cancer patients, identified by their history to be at high risk for osteoporosis, should be evaluated by BMD measurements (Hillner et al 2003). The high risk factors for breast cancer women include age $>65$ years, age 60-64 years with other risk factors (eg, family history, body weight $<70 \mathrm{~kg}$, prior non-traumatic fracture) postmenopausal women of any age receiving AIs, premenopausal women with therapy associated premature menopause. All women with breast cancer at high risk, independently of BMD results at baseline, need to repeat the BMD measurement after 1 year. The women at low risk need to be clinically monitored annually for risk status. Therefore, all postmenopausal women treated with AIs can be considered at high risk and evaluated for BMD annually. In the presence of osteoporosis (BMD $\mathrm{T}$ score $<-2.5)$ the patients should have pharmacologic therapy initiated with an antiosteoporotic drug. A calcium vitamin D supplementation is also recommended for all women treated with AIs.

Although there is currently no approved treatment or any prevention therapy for the aromatase-inhibitor induced bone loss, clinical trial evidence indicates that intravenous and oral bisphosphonates are effective in maintaining bone density in breast cancer patients on hormone therapy and with therapy-associated premature menopause. In particular iv bisphosphonate zoledronic acid has shown clinical benefits in the treatment of bone metastases among patients with solid tumors. In a Cochrane review that assessed all approved oral and iv bisphosphonates for breast cancer treatment, 
zoledronic acid produced a greater reduction in the risk of skeletal related events with respect to both placebo $(-41 \%)$ and ibandronate, clodronate and pamidronate (ranging from $-14 \%$ to $-23 \%$ ) (Pavlakis et al 2005).

A recent substudy of the Austrian Breast and Colorectal Cancer Study Group (ABCSG) has demonstrated that zoledronic acid (4 mg iv every 6 months over 3 years) was effective in counteracting bone loss in premenopausal women receiving adjuvant endocrine therapy (goserelin plus anastrozole) for hormone-responsive breast cancer (Gnant et al 2007).

In the Zometa-Femara Adjuvant Synergy Trial (Z-FAST), zoledronic acid also appeared to prevent bone loss in postmenopausal women with stage I-IIIa estrogen and/or progesterone-receptor positive breast cancer receiving adjuvant letrozole $(n=602)$ (Brufsky et al 2007). Three hundred and one of those patients received upfront zoledronic acid (4 mg iv every 6 months), whereas the other 301 women in the delayed arm of the trial received zoledronic acid only if their T-score at lumbar spine or total hip decreased below $-2.0 \mathrm{SD}$ or if they had a clinical fracture. After 1 year of zoledronic acid treatment ( $4 \mathrm{mg}$ iv every 6 months), the lumbar spine and total hip BMD increased by $2.0 \%$ and $1.4 \%$ respectively. In contrast, among women in the delayed arm of the trial, BMD decreased substantially by $2.6 \%$ and $2.1 \%$ at lumbar spine and total hip, respectively (Brufsky et al 2007).

Despite these promising data, the use of an aggressive preventive strategy using iv bisphosphonates needs to be validated by larger randomized studies. In fact in recent years there has been an increasing number of reports which suggest that the prolonged use of intravenous bisphosphonates may be associated with osteonecrosis of the jaw (ONJ), an uncommon manifestation characterized by the presence of exposed bone in the oral cavity (Weitzam et al 2007; Brufsky 2008).

A retrospective analysis in Australia (2004-2005) reported a $0.88 \%$ to $1.15 \%$ frequency of ONJ among patients with malignant bone disease from cancer and a frequency of only $0.01 \%$ to $0.04 \%$ among patients with osteoporosis receiving a bisphosphonate. Therefore, the frequency of ONJ appears to be lower among patients with no malignant bone disease compared with patients who have advanced cancer (Mavrokokki et al 2007).

A possible alternative in the prevention of bone loss in breast cancer women is represented by oral bisphosphonates, which are less commonly associated with the development of osteonecrosis of the jaw than intravenous bisphosphonates.
It has recently been reported that oral bisphosphonate risedronate prevents anastrozole induced bone loss with an increase of BMD at lumbar spine and a decrease of bone turnover markers (Confavreux et al 2007).

In conclusion the negative effects of AIs on bone have to be taken into account and all women should receive lifestyle advice. Aromatase inhibitor-associated bone loss may represent a preventable and curable condition, and in high risk women a treatment with antiresorptive agents, such as bisphosphonates, should be considered.

\section{Effects of Als on lipids}

Large-scale epidemiological studies have shown that high serum levels of total cholesterol (TC) and LDL-cholesterol (LDL-C) are important risk factors for the development of cardiovascular diseases (CVD), and that low serum levels of HDL-cholesterol (HDL-C) and hypertriglyceridemia (TG) are associated with increased coronary heart diseases (CHD), morbidity and mortality. New emerging risk factors for $\mathrm{CHD}$, including insulin resistance, glucose intolerance and prothrombotic state, are an issue of growing interest in CVD prevention. Estrogens have a protective effect on the lipid profile in human subjects, and high levels of HDL-C and low levels of LDL-C are associated with high estrogen levels. Therefore, the reduction of estrogen levels which occur during adjuvant therapy for breast cancer may lead to a more atherogenic lipid profile and increased CHD risk.

The rates of CHD in women after the menopause are 2 to 3 times those of premenopausal women of the same age and the dramatic decline in estrogen levels during the menopause is associated with unfavorable changes in lipid profile (Rosano et al 1996). Estrogen replacement therapy is known to have a mixed effect on serum lipids, resulting in a significant decrease in TC and LDL-C, a favorable increase in HDL-C, but an unfavorable increase in TG. Randomized controlled trials have demonstrated that estrogen replacement therapy causes either no benefit or an increased cardiovascular risk (Manson et al 2003).

Tamoxifen has demonstrated a favorable effect on lipid profile in postmenopausal women with a reduction in TC and LDL-C levels. After 5 years of treatment with tamoxifen there was a significant change in lipid profile in node-negative postmenopausal women compared with untreated patients: TC, LDL-C and lipoprotein(a) decreased, while HDL-C remained unchanged (Love et al 1994). An adjuvant study showed that after 15 months of tamoxifen treatment there was a significant increase in serum TG levels compared with baseline levels 
(Liu and Yang 2003). With regard to AIs, current data do not allow the drawing of any clear conclusions about the effect of these drugs on lipid metabolism.

Data on lipid profile were not systematically collected in the ATAC trial; however, the prevalence of low grade hypercholesterolemia was reported to be 2.6 fold higher in patients receiving anastrozole than in those taking tamoxifen (ATAC Trialists' Group 2005).

Most studies on anastrozole in postmenopausal women with early breast cancer have shown beneficial increases in HDL-C and favorable decreases in TG, while variable effects on the levels of TC or LDL-C were described (Sawada and Sato 2003). In the ITA Trial, the switching to anastrozole after 2-3 years of tamoxifen was also associated with an increased incidence of lipid metabolism disorders $(9.3 \%$ vs $4.0 \%, p=0.04)$ with respect to patients receiving 5 years of tamoxifen treatment (Boccardo et al 2006).

Even the ARNO 95/ABSCG-8 combined analysis did not report hypercholesterolemia in women switching to 3 years of anastrozole after 2 years of tamoxifen compared to the standard 5 years of tamoxifen (Jakesz et al 2005).

With regard to letrozole, this agent caused a deterioration of lipid profile with an increase of atherogenic risk ratios TC/ HDL-C and LDL-C/HDL-C in postmenopausal women with metastatic breast cancer previously treated with tamoxifen (Elisaf et al 2001). Instead the MA-17 trial did not document any detrimental effect of letrozole on lipid levels, and similar frequencies of hypercholesterolemia were observed in the placebo and letrozole groups (Goss et al 2005).

The BIG 1-98 trial reported a greater than two-fold increase in the incidence of hypercholesterolemia with letrozole therapy compared with the tamoxifen (43.6\% vs $19.2 \%$, respectively); however, over $80 \%$ of these were mild and did not require treatment (Thurlimann et al 2005). Notably, these data were based on single, non-fasting measurement of blood cholesterol, and any single event at any time during the study resulted in a positive report. For this reason, a valid interpretation of these data is difficult.

The other AI exemestane has shown a significant reduction of TC and TG, and an unfavorable decrease in HDL-C in a small study on postmenopausal women with advanced breast cancer (Engan et al 1995). Another study showed only a significant reduction in HDL-C $(\mathrm{p}<0.001)$ and apolipoproteina A1 $(\mathrm{p}=0.004)$ in women assigned to exemestane with respect to the placebo group (Lonning et al 2005).

Exemestane has also been shown to have no detrimental effects on serum lipids or atherogenic risk in postmenopausal women with metastatic breast cancer (Atalay et al 2004).
More recently, a comparison of the effect of exemastane versus tamoxifen on the lipid profile of postmenopausal early breast cancer patients was reported in the preliminary results of the TEAM Greeck substudy at a 12 month follow-up (Markopoulos et al 2005). In this study, although mean LDL-C levels were higher in the exemestane versus the tamoxifen group, triglyceride levels were lower, while no difference was reported in total cholesterol or in HDL cholesterol. These data seem to suggest that exemestane may have a less unfavorable effect on lipids with respect to the non-steroidal aromatase inhibitors. In contrast, a recent adjuvant study compared postmenopausal women switched to exemestane treatment after 2-3 years of tamoxifen with the women who continued the 5-year tamoxifen therapy. The exemestane treated patients presented a significant $(\mathrm{p}<0.01)$ decrease in HDL-C and TG and a significant increase in serum LDL-C, whereas the women who continued tamoxifen did not show any significant changes in lipid parameters (Montagnani et al 2007). In this latter study, however, the increase in LDL-C could mainly be explained by the loss of the positive action of tamoxifen; in fact in the second year of the study, no further increase in LDL-C was observed (Montagnani et al 2007).

The effects of AIs on body composition in postmenopausal women with breast cancer, switched from adjuvant tamoxifen to adjuvant exemestane, were also investigated: fat mass significantly decreased by month 12 in the exemestane group, but not in the tamoxifen group; the between-group difference was statistically significant ( $\mathrm{p}<0.01$ ) (Francini et al 2006). In this latter study the fat free-mass/fat mass ratio significantly ( $\mathrm{p}<0.05)$ increased in the exemestane group, but not in the tamoxifen group. These findings suggest that switching patients to adjuvant exemestane treatment after at least two years of tamoxifen may be associated with an advantage over continuing adjuvant tamoxifen in terms of body composition. Since it is known that exemestane and the other AIs reduce circulating estrogen levels, and estrogens have direct effects on adipocytes, the authors suggested there may be an association between exemestane use, reduced circulating estrogen levels and body weight changes (Francini et al 2006).

Overall, the available studies suggest that the differences in lipid profile between tamoxifen and AIs may be due to the lipid-lowering capacity of tamoxifen rather than to increases in lipid levels due to AIs. However, the true difference in changes of lipid parameters between patients receiving tamoxifen and AIs still has to be defined and therefore no exact recommendation concerning the monitoring of lipid metabolism may at present be given. As a general guideline, 
patients should perform a baseline and periodic monitoring of the fasting lipid profile before starting adjuvant hormonal therapy, maintain a correct lifestyle with a healthy diet and regular exercise, and assume medications useful for the treatment of pre-existing hypercholesterolemia.

\section{Effects of Als on cardiovascular events}

Even though lipids are important risk factors, the relationship between hormone related changes in lipid profile and the development of CVD events is unclear. Although most of the studies demonstrated that tamoxifen has a favorable effect on lipid profile, this did not seem to translate into a beneficial effect on the development and progression of CVD. A meta-analysis reported that tamoxifen significantly decreased myocardial infarction deaths but did not significantly reduce the myocardial infarction incidence (Braithwaite et al 2003). The 15-year survival update from the Early Breast Cancer Trialists Collaborative group meta-analysis found a borderline significance regarding reduced mortality from heart disease in breast cancer patients receiving tamoxifen compared with a control group (120 vs $132 \mathrm{p}=0.06$ ) (Clarke 2006).

The effect of AIs on cardiovascular risk is less defined, since long-term studies are needed to evaluate the development of cardiovascular events as a consequence of treatment with these agents. Some data have been drawn from studies using anastrozole and letrozole in the advanced setting. In fact anastrozole-treated patients showed a significant decrease in thromboembolic events, including venous thromboembolism, coronary ischemic and cerebrovascular events compared with tamoxifen (Nabholtz et al 2000; Mouridsen et al 2001). In the ATAC study, anastrozole resulted in a clinically significant reduction in the incidence of cerebrovascular events $(2.0 \%$ vs $2.8 \%$ ) and thromboembolic events ( $2.0 \%$ vs $4.5 \%)$ in comparison with tamoxifen (ATAC Trialists' Group 2005). In the same study a non significant increase in ischemic cardiovascular diseases was associated with anastrozole therapy. Data on cardiovascular diseases were not reported in detail in the ABCSG/ARNO Trial, but there was no difference in the incidence of myocardial infarction between the anastrozole arm and the tamoxifen arm (Jakesz et al 2005). The overall reported incidence of cardiovascular events in BIG 1-98 was similar in the two groups (5.5\% and $5 \%$ for letrozole and tamoxifen respectively) (Coates et al 2007). However, in BIG 1-98 there was an excess of severe events (grades 3-5) of both heart failure and ischemic heart disease in the letrozole arm with respect to the tamoxifen arm $(1.0 \% \mathrm{vs}$ $0.6 \%$ and $2.2 \%$ vs $1.7 \%$, respectivaly) (Coates et al 2007 ).
A recent reanalysis of the cardiovascular adverse events of BIG 1-98 trial has reported that at a median follow-up time of 30.1 months there was a similar overall incidence of cardiac adverse events (letrozole 4.8\%; tamoxifen 4.7\%) but more grade 3 to 5 cardiac adverse events on letrozole (letrozole 2.4\%; tamoxifen 1.4\%; p < 0.001) and more overall tromboembolic events on tamoxifen (tamoxifen 3.9\%; letrozole 1.7\%; $<<0.001$ ) (Mouridsen et al 2007).

The effect of prolonging letrozole treatment beyond 5 years is currently being addressed in the MA.17R rerandomization study in which patients who remain disease-free after completing 5 years of letrozole extended adjiuvant therapy are randomized to letrozole or placebo for a further 5 years. This study will provide further data on safety issues associated with long-term use of letrozole (Goss et al 2003; Goss et al 2005).

A non-significant increase in the incidence of cardiovascular disease was also reported in patients switching to exemestane in the IES trial, including a 2.5-fold increase in the number of myocardial infarctions (Coombes et al 2007).

However, in this trial, as well as other postadjuvant or switching studies, it is difficult to deduce whether the cardiovascular adverse events observed may be attributed, at least in part, to the effects of prior tamoxifen or whether these events are a true effect of the AIs.

Overall, the current available findings suggest that the impact on CVD of the long-term use of AIs is still to be clarified. Nevertheless, in the above mentioned trials definitions of cardiac and ischemic disease were not consistently defined, follow-up was short ( $<10$ years), and risk factors for coronary disease (including lipid profiles) were not systematically documented. However, when starting a treatment with AIs it is important to determine the presence of associated comorbidities, such as diabetes mellitus, hypertension, smoking, and/or the use of anthracycline-based chemotherapy, which may increase the risk of developing cardiomyopathy and symptomatic heart failure or other cardiovascular events. Therefore, when treating breast cancer patients, oncologists should correct cardiovascular risk factors as is done with patients who do not have breast cancer. Moreover, physicians should assess cardiovascular risk, monitor and treat patients already diagnosed at risk for coronary heart disease, according to established guidelines (Winer et al 2004; Ponzone et al 2008). Another point to consider is that the relationship between lipid profiles, hormonal status, and cardiovascular risk is not always clear. Lipids, as risk factors, are intermediate end points and may not correlate with the clinically relevant end points of the incidence of 
cardiovascular events in long-term studies. To sum up, AIs are associated with lower thrombotic risk when compared with tamoxifen, while the possible unfavorable balance of AIs on cardiovascular risk needs to be confirmed.

\section{Effects of Als on the musculoskeletal system}

Clinical experience suggests that the treatment with AIs is associated with a novel musculoskeletal side effect consisting of an arthralgia syndrome which sometimes can be a reason for discontinuation of AIs treatment. Arthralgia is defined as pain or stiffness in the joints, which in patients treated with AIs is not caused by arthritis, the typical onset of AIs associated arthralgia is within 2 months of treatment initiation (Burstein 2007). All the major adjuvant trials of AIs have reported the incidence of musculoskeletal symptoms or asked directly about arthralgia. Therefore, the reported incidence of arthralgia syndrome reflects different definitions of symptoms and is markedly different in individual studies. In fact in the ATAC trial there was a significant increase in arthralgias in the anastrozole arm when compared to the tamoxifen arm $(35.6 \%$ vs $29.4 \%$; p $<0.001)$ (ATAC Trialists' Group 2005). Also, MA 17 trial reported an increased frequency of arthralgias in women treated with letrozole with respect to those in the placebo arm $(25.3 \%$ vs $20.6 \%$; $<0.001$ ) (Goss et al 2005). Finally, the IES study confirmed that arthralgias were more frequent in the exemestane group (20.6\%) (Coombes et al 2007). Even though the true etiology of AIs-associated arthralgia syndrome is not known, several studies have shown a link between low serum levels of estrogen and arthralgias. The natural hypoestrogenemia of menopause is also associated with arthralgia, which can be ameliorated with hormone replacement therapy (HRT) or exacerbated with AIs treatment. Moreover, it is known that estrogens regulate inflammatory cytokines and enhance nociception at the level of the central nervous system. Although it is clear that AIs worsen arthralgias compared with either placebo or tamoxifen, the overall impact on quality of life and associated morbidity still needs to be determined (Burstein 2007; Coleman et al 2008).

\section{Conclusions}

The third-generation aromatase inhibitors (AIs), letrozole, anastrozole and exemestane, are now widely accepted as optimal adjuvant treatment for postmenopausal women with early breast cancer. This means that individual patients will be exposed to these agents for longer durations, making it increasingly important to establish their long-term safety.
This review focused on the effects of AIs on bone metabolism, lipid profile, and CVD. Despite the lack of comparative studies, current data suggest that all the third generation AIs may have adverse effects on bone, with reduction in BMD and an increase in the rate of bone fractures. However, aromatase inhibitor-associated bone loss may represent a preventable and curable condition, and a treatment with antiresorptive agents, such as bisphosphonates, should be considered in high risk women. With respect to tamoxifen AIs present lower thrombotic risk but a less favorable impact on lipid profile. However, the true impact on CVD of the long-term use of AIs still remains to be clarified. Even though experimental and clinical data suggest that different AIs may have a different impact on fracture and cardiovascular risk, at present, no consensus exists as to whether the best AI for individual patients could be chosen.

To sum up, as a general guideline, it may be recommended that patients undergo BMD and lipid profile evaluation before and during adjuvant therapy with AIs, maintain a correct lifestyle with a healthy diet and regular exercise, and assume medications useful for managing pre-existing osteoporosis and hypercholesterolemia.

\section{Disclosures}

The authors have no conflicts of interest to disclose.

\section{References}

The Arimidex, Tamoxifen, Alone or in Combination (ATAC) Trialists' Group 2005. Results of the ATAC (arimidex, tamoxifen, alone or in combination) trial after completion of 5 years' adjuvant treatment for breast cancer. Lancet, 365:60-2.

Arimidex, Tamoxifen, Alone or in Combination (ATAC) Trialists' Group 2008. Effect of anastrozole and tamoxifen as adjuvant treatment for early-stage breast cancer: 100-month analysis of the ATAC trial. Lancet Oncol, 9:45-53.

Atalay G, Dirix L, Biganzoli L, et al. 2004. The effect of exemestane on serum lipid profile in postmenopausal women with metastatic breast cancer: a companion study to EORTC Trial 10951, Randomized phase II study in first line hormonal treatment for metastatic breast cancer with exemestane or tamoxifen in postmenopausal patients. Ann Oncol, 15:211 -7.

Bajetta E, Zilembo N, Bichisao E. 2002. Endocrine effects of nonsteroidal aromatase inhibitors and their clinical impact. J Clin Oncol, 20:3039-40.

Baum M, Buzdar AU, Cuzick J, et al. 2002. Anastrozole alone or in combination with tamoxifen versus tamoxifen alone for adjuvant treatment of postmenopausal women with early breast cancer: first results of the ATAC randomized trial. Lancet, 359:2131-9.

Boccardo F, Rubagotti A, Guglielmini P, et al. 2006. Switching to anastrozole versus continued tamoxifen treatment of early breast cancer. Updated results of the Italian tamoxifen anastrozole (ITA) trial. Ann Oncol, 17:10-4.

Bonneterre J, Buzdar A, Nabholtz JM, et al. 2001. Anastrozole is superior to tamoxifen as first-line therapy in hormone-receptor positive advanced breast carcinoma. Cancer, 92:2247-58.

Braithwaite RS, Chlebowski RT, Lau J, et al. 2003. Meta-analysis of vascular and neoplastic events associated with tamoxifen. J Gen Intern Med, 18:937-74. 
Bria E, Ciccarese M, Giannarelli D, et al. 2006. Early switch with aromatase inhibitors as adjuvant hormonal theraphy for postmenopausal breast cancer: pooled-analysis of 8794 patients. Cancer Treat Rev, 32:325-32.

Brufsky A, Marker WG, Beck JT, et al. 2007. Zoledronic acid inhibits adjuvant letrozole-induced bone loss in postmenopausal women with early breast cancer. J Clin Oncol, 25:828-36.

Brufsky A.M. 2008. Cancer treatment induced bone loss: pathophysiology and clinical perspectives. The Oncologist, 13:187-95.

Burstein HJ. 2007. Aromatase inhibitor-associated arthralgia syndrome. Breast, 16:223-34.

Buzdar A, Howell A. 2001. Advances in aromatase inhibition: clinical efficacy and tolerability in the treatment of breast cancer. Clin Cancer Res, 7:2620-35.

Carlini P, Bria E, Ferretti G, et al. 2005. New aromatase inhibitors (Ais) as first-line endocrine therapy (ET) in metastatic breast cancer (MBC): A pooled analysis of 3238 women from 8 phase III trials. ASCO 2005, 23:602.

Cataliotti L, Buzdar AU, Noguchi S, et al. 2006. Comparison of anastrozole versus tamoxifen as pre-operative therapy in postmenopausal women with hormone receptor-positiev breast cancer: the pre-operative 'Arimidex' compared to tamoxifen (PROACT) trial. Cancer, 106:2095-103.

Clarke M. 2006. Meta-analyses of adjuvant therapies for women with early breast cancer: the Early Breast Cancer Trialists' Collaborative Group overview. Ann Oncol, 17:59-62.

Coates AS, Keshaviah A, Thulimann B, et al. 2007. Five years of letrozole compared with tamoxifen as initial adjuvant therapy for postmenopausal women with endocrine-responsive early breast postmenopausal women with endocrine-responsive early breast cancer: update of study BIG 1-98. J Clin Oncol, 25:486-92.

Coleman RE, Banks LM, Girgis SI, et al. 2007. Skeletal effects of exemestane on bone-mineral density, bone biomarkers, and fracture incidence in postmenopausal women with early breast cancer participating in the Intergroup Exemestane Study (IES): a randomised controlled study. Lancet Oncol, 8:119-27.

Coleman RE, Bolten WW, Lansdown M, et al. 2008. Aromatase inhibitorinduced arthralgia: clinical experience and treatment recommendations. Cancer Treat Rev, 34:275-82.

Confavreux C, Fontana A, Guastalla JP, et al. 2007. Estrogen-dependent increase in bone turnover and bone loss in postmenopausal women with breast cancer treated with anastrozole. Prevention with bisphosphonates. Bone, 41:346-52.

Coombes RC, Kilburn LS, Snowdon CF, et al. 2007. Survival and safety of exemestane versus tamoxifen after 2-3 years' tamoxifen treatment (Intergroup Exemestane Study): a randomised controlled trial. Lancet, 369:559-70.

Cummings SR, Browner WS, Bauer D, et al. 1998. Endogenous hormones and the risk of hip and vertebral fractures among older women. $N$ Engl JMed, 339:733-8.

Cuzick J, Powles T, Veronesi U, et al. 2003. Overview of the main outcomes in breast-cancer prevention trials. Lancet, 361:296-300.

Eastell R, Hannon RA, Cuzick J, et al. 2006. Effects on an Aromatase inhibitor on BMD and bone turnover markers: 2-year results of the anastrozole, tamoxifen, alone or in combination (ATAC) trial. J Bone Miner Res, 21:1215-23.

Elisaf MS, Bairaktari ET, Nicolaides C, et al. 2001. Effect of letrozole on the lipid profile in postmenopausal women with breast cancer. Eur $J$ Cancer, 37:1510-3.

Engan T, Krane J, Johannessen DC, et al. 1995. Plasma changes in breast cancer patients during endocrine therapy - lipid measurements and nuclear magnetic resonance (NMR) spectroscopy. Breast Cancer Res Treat, 36:287-97.

Francini G, Petrioli R, Montagnani A, et al. 2006. Exemestane after tamoxifen as adjuvant hormonal therapy in postmenopausal women with breast cancer: effect on body composition and lipid. Br J Cancer, 95:153-8.
Ganz PA. 2001. Impact of tamoxifen adjuvant therapy on symptoms, functioning, and quality of life. J Natl Cancer Inst, 30:130-4.

Gnant MF, Mlineritsch B, Luschin-Ebengreuth G, et al. 2007. Zoledronic acid prevents cancer treatment-induced bone loss in premenopausal women receiving adjuvant endocrine therapy for hormone responsive breast cancer: a report from Austrian Breast and Colorectal Cancer Study Group. J Clin Oncol, 25:820-8.

Gonnelli S, Cadirni A, Caffarelli C, et al. 2007. Changes in bone turnover and in bone mass in women with breast cancer switched from tamoxifen to exemestane. Bone, 40:205-10.

Goss PE, Ingle JN, Martino S, et al. 2003. A randomized trial of letrozole in postmenopausal women after five years of tamoxifen for early-stage breast cancer. N Eng J Med, 349:1793-802.

Goss PE, Ingle JN, Martino S, et al. 2005. Randomized trial of letrozole following tamoxifen as extended adjuvant therapy in receptor positive breast cancer; updated findings from NCIC CTG MA.17. J Natl Cancer Inst, 97:1262-71.

Goss PE, Hadji P, Subar M, et al. 2007. Effects of steroidal and nonsteroidal aromatase inhitors on markers of bone turnover in healthy postmenopausal women. Breast Cancer Res, 9:R52.

Heshmati HM, Khosla S, Robins SP, et al. 2002. Role of low levels of endogenous estrogen in regulation of bone resorption in late postmenopausal women. J Bone Miner Res, 17:172-8.

Hillner BE, Ingle JN, Chlebowski RT, et al. 2003. American Society of Clinical Oncology 2003 update on the role of bisphosphonates and bone health issues in women with breast cancer. J Clin Oncol, 21:4042-4057.

Jakesz R, Jonat W, Gnant M, et al. 2005. Switching of postmenopausal women with endocrine-responsive early breast cancer to anastrozole after 2 years' adjuvant tamoxifen: combined results of ABCSG trial 8 and ARNO 95 trial. Lancet, 366:455-62.

Jakesz R, Greil R, Gnant M, et al. 2007. Extended adjuvant therapy with anastrozole among postmenopausal breast cancer patients: results from the randomized Austrian Breast and Colorectal Cancer Study Group Trial 6a. J Natl Cancer Inst, 19:1845-53.

Jordan VC 2007. New insights into the metabolism of tamoxifen and its role in the treatment and prevention of breast cancer. Steroids, $72: 829-42$.

Journé F, Body JJ, Leclercq G, et al. 2008. Hormone therapy for breast cancer, with an emphasis on the pure antiestrogen fulvestrant: mode of action, antitumor efficacy and effects on bone health. Expert Opin Drug Saf, 7:241-58.

Kaufmann M, Jonat W, Hilfrich J, et al. 2007. Improved overall survival in postmenopausal women with early breast cancer after anastrozole initiated after treatment with tamoxifen compared with continued tamoxifen: the ARNO 95 Study. J Clin Oncol, 25:2664-70.

Liu CL, Yang TL. 2003. Sequential changes in serum triglyceride levels during adjuvant tamoxifen therapy in breast cancer patients and the effect of dose reduction. Breast Cancer Res Treat, 79:11-6.

Lonning PE, Geisler J, Krag LE, et al. 2005. Effects of exemestane administred for 2 years versus placebo on bone mineral density, bone biomarkers, and plasma lipids in patients with surgically resected early breast cancer. J Clin Oncol, 23:5126-37.

Love RR, Wiebe DA, Feyzi JM, et al. 1994. Effects of tamoxifen on cardiovascular risk factors in postmenopausal women after 5 years of treatment. J Natl Cancer Inst, 86:1534-9.

Mavrokokki T, Cheng A, Stein B, et al. 2007. Nature and frequency of bisphosphonates-associated osteonecrosis of the jaws in Australia. J Oral Maxillofac Surg, 65:415-23.

Manson JE, Hsia J, Johnson KC, et al. 2003. Estrogen plus progestin and the risk of coronary heart disease. $N$ Engl J Med, 349:523-34.

Markopoulos C, Polychronis A, Zobolas V, et al. 2005. The effect of exemestane on the lipidemic profile of postmenopausal early breast cancer patients: preliminary results of the TEAM Greek sub-study. Breast Cancer Res Treat, 93:61-6.

McCloskey E. 2006. Effects of third-generation aromatase inhibitors on bone. Eur J Cancer, 42:1044-51. 
Mincey BA, Duh MS, Thomas SK, et al. 2006. Risk of cancer treatment-associated bone loss and fractures among women with breast cancer receiving aromatase inhibitors. Clin Breast Cancer, 7:127-32.

Montagnani A, Gonnelli S, Cadirni A, et al. 2007. The effects on lipid serum levels of a 2-year adjuvant treatment with exemestane after tamoxifen in postmenopausal women with early breast cancer. in press 10.1016/j.ejim.2007.05.016

Mouridsen H, Gershanovich M, Sun Y, et al. 2001. Superior efficacy of letrozole versus tamoxifen as first-line therapy for postmenopausal women with advanced breast cancer: results of a phase III study of the International Letrozole Breast Cancer Group. J Clin Oncol, 14:2000-11.

Mouridsen H, Keshaviah A, Coates AS, et al. 2007. Cardiovascular adverse events during adjuvant endocrine therapy for early breast cancer using letrozole or tamoxifen: safety analysis of BIG 1-98 trial. J Clin Onco, 25:5715-22.

Nabholtz JM, Buzdar A, Pollak M, et al. 2000. Anastrozole is superior to tamoxifen as first-line therapy for advanced breast cancer in postmenopausal women: results of a North American multicenter randomized trial-Arimidex Study Group. J Clin Oncol, 18:3758-67.

Neven P, Paridaens R, Pelgrims G, et al. 2008. Fulvestrant (Faslodextrade mark) in advanced breast cancer: clinical experience from a Belgian cooperative study. Breast Cancer Res Treat, 109:59-65.

Pavlakis N, Schmidt RL, Stockler M. 2005. Bisphosphonates for breast cancer. Cochrance Database Syst Rev, (3):CD003474.

Perez EA, Josse RG, Pritchard KI, et al. 2006. Effect of letrozole versus placebo on bone mineral density in women with primary breast cancer completing 5 or more years of adjuvant tamoxifen: A companion study to NCIC CTG MA.17. J Clin Oncol, 24:3629-35.
Ponzone R, Mininanni P, Cassina E, et al. 2008. Aromatase inhibitors for breast cancer: different structures, same effects? Endocrine-Related Cancer, 15:27-36.

Rosano GM, Chierchia SL, Leonardo F, et al. 1996. Cardioprotective effects of ovarian hormones. Eur Heart J, 17:15-9.

Sawada S, Sato K. 2003. Effect of anastrozole and tamoxifen on serum lipid levels in Japanese postmenopausal women with early breast cancer. Breast Cancer Res Treat, 82:S31-2.

Smith IE, Dowsett M. 2003. Aromatase inhibitors in breast cancer. $N$ Engl J Med, 348:2431-42.

Thurlimann B, Keshaviah A, Coates AS, et al. 2005. A comparison of letrozole and tamoxifen in postmenopausal women with early breast cancer. N Engl J Med, 353:2747-57.

Viale G, Regan MM, Maiorano E, et al. 2007. Prognostic and predictive value of centrally reviewed expression of estrogen and progesterone receptors in a randomized trial comparing letrozole and tamoxifen adjuvant therapy for postmenopausal women with early breast cancer: results from the BIG 1-98 collaborative groups. J Clin Oncol, 25:3846-52.

Weitzman R, Sauter N, Eriksen EF, et al. 2007. Critical review: Updated recommendations fort he prevention, diagnosis, and treatment of osteonecrosis of the jaw in cancer patients-May 2006. Crit Rev Oncol Hematol, 62:148-52.

Winer EP, Hudis C, Burstein HJ, et al. 2004. American Society of Clinical Oncology technology assessment on the use of aromatase inhibitors as adjuvant therapy for postmenopausal women with hormone receptor-positive breast cancer: status report 2004. J Clin Oncol, 23:619-9. 
